\title{
Exact eigenfunctions for square-wave gratings: Application to diffraction and surface-plasmon calculations
}

\author{
Ping Sheng \\ Corporate Research Science Laboratories, Exxon Research and Engineering Company, \\ P. O. Box 45, Linden, New Jersey 07036 \\ R. S. Stepleman \\ Computing Technology Services Division, Exxon Research and Engineering Company, \\ P. O. Box 45, Linden, New Jersey 07036 \\ P. N. Sanda \\ Corporate Research Science Laboratories, Exxon Research and Engineering Company, \\ P. O. Box 45, Linden, New Jersey 07036 \\ (Received 12 April 1982)
}

\begin{abstract}
We show that for dielectric or metallic gratings with square-wave geometry the photon eigenfunctions in the grating region can be expressed in analytical form. The knowledge of these eigenfunctions makes the diffraction calculation not only simple and direct, but also devoid of the many limitations encountered in other solution techniques. In particular, diffraction from deep gratings and the calculation of surface-plasmon excitations present no difficulties. Numerical results on diffraction efficiency are in good agreement with our experimental data. The near-field electromagnetic properties of Ag gratings are examined in detail. These results are of particular importance in understanding the optical behavior of molecules near a rough metal surface (for example, in surface-enhanced Raman scattering). It is shown that coincident with the excitation of surface plasmon, there is an enhancement of the local- (surface-) field intensity by a factor of 100-500. For molecules on a grating surface, such increase in local fields can imply an amplification of $10^{4}-3 \times 10^{5}$ for the Raman scattering signal.
\end{abstract}

\section{INTRODUCTION}

Diffraction of light by periodically corrugated surfaces has been a subject of continuing interest since the early days of the electromagnetic theory. Mathematical treatment of the problem started with Kirchhoff's scalar-diffraction theory. The scalar theory ignores the vector nature of light and treats the interaction of light with the scattering object only in an approximate manner. Although satisfactory for diffraction problems where the scattering objects are much larger than the wavelength of light $\lambda$, the approximations involved in the scalar theory are known to break down ${ }^{1}$ when the size or periodicity of the diffracting object becomes comparable to or smaller than $\lambda$. Subsequent to Kirchhoff's theory, an alternative approach to diffraction calculation was advanced by Lord Rayleigh ${ }^{2}$ in his solution to the problem of wave scattering from a reflecting grating. By ex- pressing the wave field everywhere in space as superpositions of plane-wave harmonics, Rayleigh obtained the diffraction amplitudes through boundary-condition fitting at the grating surface. Rayleigh's method yields reasonable results for shallow gratings even when the periodicity is smaller than $\lambda$. However, when the ratio of grating depth to periodicity exceeds a small critical value, the method fails to converge. ${ }^{3}$ This severe constraint naturally limits the applicability of Rayleigh's method to problems involving only weak scatterings. In recent years, the increasing availability of digital computers has brought along a host of numerical techniques ${ }^{4}$ for the solution of diffraction problems. Although most of the methods are rigorous in formulation, they nevertheless exhibit different effectiveness in actual execution. For example, direct numerical solution of the wave equation in differential form is reported to show instabilities for gratings made of good 
conductors, such as aluminum or silver. ${ }^{4}$ The integral-equation approach, on the other hand, is numerically more stable than the differential method but can display matrix ill-conditioning problems when grating depth becomes too large. ${ }^{5}$

It is the purpose of this paper to point out that for the class of dielectric or metallic gratings with rectangular (square-wave) geometries, eigenfunctions of Maxwell's equations (in the region of corrugations) can be expressed in analytical form. With the knowledge of these eigenfunctions, the diffraction calculation is shown to be not only simple and direct, but also devoid of the many limitations encountered in other solution techniques. In particular, diffraction by deep gratings and the calculation of surface-plasmon excitations are noted to present no difficulties. As an application of our method, we examine the near-field electromagnetic properties of metallic gratings. It is shown that the excitation of surface plasmons on Ag gratings causes the local surface field to increase by a factor of 100-500. For molecules adsorbed on the grating surface, such local-field enhancement may account for a large part of the observed Ramansignal amplification. ${ }^{6}$

In what ensues, the eigenvalues and eigenfunctions of Maxwell's equations for square-wave gratings are considered in Sec. II. The formalism for the solution of the diffraction problem is delineated in Sec. III. In Sec. IV we present theoretical results and compare experimental data with some of the predictions. Good agreements are obtained in all cases.

\section{EIGENFUNCTIONS OF SQUARE-WAVE GRATINGS}

Figure 1 gives the coordinate system to be used in the calculation. The top of the grating is at the plane $z=0$. The periodicity of the grating is denoted by $d$, the depth by $h$, and the ratio of the grating linewidth to periodicity by $r$. We identify two polarizations for the plane wave incident at the angle $\theta_{i}: E(H)$ polarization is defined as the configuration in which the electric (magnetic) vector of the light is parallel to the $y$ axis. To obtain

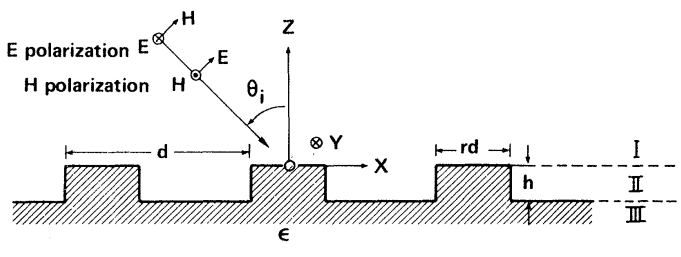

FIG. 1. Geometry of the rectangular grating used in the calculation.

the solution of the diffraction problem, let us consider the form of the wave field in each of the three regions separated by the planes $z=0$ and $z=h$ as shown in Fig. 1. In regions $I$ and III, the electromagnetic field can be expressed as superposition of plane and evanascent waves:

$$
\begin{aligned}
\Psi^{\mathrm{I}}= & \exp \left[-i k_{0}\left(\sin \theta_{i} x+\cos \theta_{i} z\right)\right] \\
& +\sum_{n=-\infty}^{\infty} R_{n} \exp \left\{i k_{0}\left[\gamma_{n} x+\left(1-\gamma_{n}^{2}\right)^{1 / 2} z\right]\right\}, \\
\Psi^{\mathrm{III}}= & \sum_{n=-\infty}^{\infty} T_{n} \exp \left\{i k_{0}\left[\gamma_{n} x-\left(\epsilon-\gamma_{n}^{2}\right)^{1 / 2} z\right]\right\}
\end{aligned}
$$

Here $\Psi$ is the $y$ component of the magnetic (electric) vector for the $H(E)$-polarized wave, $k_{0}=2 \pi / \lambda$ is the free-space wave vector of light, $\epsilon$ is the complex dielectric constant of the grating, $R_{n}$ and $T_{n}$ are the amplitudes of the $n$th reflected and trasmitted diffraction orders, respectively, and $\gamma_{n}$ is defined as

$$
\gamma_{n}=\sin \theta_{i}+\frac{n \lambda}{d}
$$

In Eqs. (1) and (2), and in the rest of this paper, the square root with the positive imaginary part will always be chosen.

In region II the space is no longer homogeneous, and the solution of the wave equation generally requires more effort. However, in the particular instance where the grating is perfectly conducting, the field can again be written down simply. Noting the requirement that the tangential component of the electric field must vanish on the grating surface, we have, for the $E$ polarization, ${ }^{7}$

$$
E_{y}^{\mathrm{II}}=\left\{\begin{array}{l}
\sum_{m=1}^{\infty} A_{m} \sin \left[\frac{m \pi}{a}\left[x+\frac{a}{2}\right]\right] \sin \left\{k_{0}\left[1-\left[\frac{m \lambda}{2 a}\right]^{2}\right]^{1 / 2}(z+h)\right\}, \quad|x|<\frac{a}{2} \\
0, \frac{a}{2}<|x|<\frac{d}{2} .
\end{array}\right.
$$


Here $a=(1-r) d$ is the width of the rectangular channel. Similarly, for the $H$ polarization, $H_{y}^{\text {II }}$ is given by ${ }^{7}$

$$
H_{y}^{\mathrm{II}}=\left\{\begin{array}{l}
\sum_{m=0}^{\infty} B_{m} \cos \left[\frac{m \pi}{a}\left[x+\frac{a}{2}\right]\right] \cos \left\{k_{0}\left[1-\left[\frac{m \lambda}{2 a}\right]^{2}\right]^{1 / 2}(z+h)\right\}, \quad|x|<\frac{a}{2} \\
0, \frac{a}{2}<|x|<\frac{d}{2}
\end{array}\right.
$$

Equations (4a) and (4b), plus the condition $\Psi^{\mathrm{III}}=0$, have been the basis of much of the theoretical work involving perfectly conducting rectangular gratings. The diffraction calculation in those cases involves just the solution of a set of linear simultaneous equations obtained from fitting the proper boundary conditions at $z=0$, and the solutions are known to be numerically stable. For dielectric and metallic gratings, we show below that although the eigenfunctions in region II are more complicated than those of the perfectly conducting grating, they nevertheless can be expressed in closed form.

Consider Maxwell's equations for the $H$ polarization:

$$
\begin{aligned}
E_{x} & =-\frac{i}{k_{0} \epsilon(x)} \frac{\partial H_{y}}{\partial z}, \\
E_{z} & =\frac{i}{k_{0} \epsilon(x)} \frac{\partial H_{y}}{\partial x}, \\
H_{y} & =\frac{i}{k_{0}}\left(\frac{\partial E_{z}}{\partial x}-\frac{\partial E_{x}}{\partial z}\right),
\end{aligned}
$$

where $\epsilon(x)=\epsilon$ for $|x-n d| \leq|r d / 2|$, and 1 otherwise. Substitution of Eqs. (5a) and (5b) into Eq. (5c) yields

$$
\frac{\partial^{2} H_{y}}{\partial z^{2}}+\frac{\partial^{2} H_{y}}{\partial x^{2}}+\epsilon(x) k_{0}^{2} H_{y}=\frac{\partial H_{y}}{\partial x} \frac{d}{d x}[\ln \epsilon(x)] .
$$

By writing $H_{y}(x, z)=X(x) Z(z)$, Eq. (6) can be separated into two equations for $X$ and $Z$. If we denote the separation variable as $\Lambda^{2}$, then

$$
\begin{aligned}
& \frac{d^{2} Z}{d z^{2}}=-\Lambda^{2} Z \\
& \frac{d^{2} X}{d x^{2}}-\frac{d}{d x}[\ln \epsilon(x)] \frac{d X}{d x}+\left[\epsilon(x) k_{0}^{2}-\Lambda^{2}\right] X=0 .
\end{aligned}
$$

Equation (7a) can be directly solved:

$$
Z(z) \propto \exp (i \Lambda z) .
$$

However, to get $X$, we will turn to Eqs. (5b) and (5c) instead of to Eq. (7b). That is,

$$
\begin{aligned}
& \frac{d X}{d x}=-i k_{0} \epsilon(x) V, \\
& \frac{d V}{d x}=-i k_{0}\left(1-\frac{\Lambda^{2}}{\epsilon(x) k_{0}^{2}}\right) X,
\end{aligned}
$$

where we have expressed $E_{z}$ as $V(x) \exp (i \Lambda z)$, and $H_{y}$ as $X(x) \exp (i \Lambda z)$. At this stage our problem becomes identical ${ }^{8}$ to that of a periodically stratified medium with a piecewise constant $\epsilon(x)$. This exact correspondence enables us to utilize the wellknown fact that the values of $X$ and $V$ at any two values of $x$ can be related by a $2 \times 2$ transmission matrix. In our case,

$$
\left[\begin{array}{l}
X\left(-\frac{r d}{2}\right) \\
V\left(-\frac{r d}{2}\right)
\end{array}\right]=\left[\begin{array}{cc}
\cos (\beta r d) & -i \frac{\epsilon k_{0}}{\beta} \sin (\beta r d) \\
-i \frac{\beta}{\epsilon k_{0}} \sin (\beta r d) & \cos (\beta r d)
\end{array}\right]\left[\begin{array}{c}
X\left(\frac{r d}{2}\right) \\
V\left(\frac{r d}{2}\right)
\end{array}\right],
$$

where $\beta=\left(\epsilon k_{0}^{2}-\Lambda^{2}\right)^{1 / 2}$. Now since $X(r d / 2), V(r d / 2)$ represent the tangential $H$ and $E$ fields at the grating-air interface, by the electromagnetic boundary conditions they must exhibit continuity. Therefore, we can further relate $X((1-r / 2) d), V((1-r / 2) d)$ to $X(r d / 2), V(r d / 2)$, by 


$$
\left[\begin{array}{l}
X\left(\frac{r d}{2}\right) \\
V\left(\frac{r d}{2}\right)
\end{array}\right]=\left[\begin{array}{cc}
\cos [\alpha(1-r) d] & -i \frac{k_{0}}{\alpha} \sin [\alpha(1-r) d] \\
-i \frac{\alpha}{k_{0}} \sin [\alpha(1-r) d] & \cos [\alpha(1-r) d]
\end{array}\right]\left[\begin{array}{c}
X\left(\left[1-\frac{r}{2}\right) d\right] \\
\left.V\left(\mid 1-\frac{r}{2}\right) d\right]
\end{array}\right],
$$

where $\alpha=\left(k_{0}^{2}-\Lambda^{2}\right)^{1 / 2}$. Substituting Eq. (11) into Eq. (10) and observing that ${ }^{9}$

$$
\left[\begin{array}{l}
\left.X\left(1-\frac{r}{2}\right) d\right] \\
V\left(\left[1-\frac{r}{2}\right) d\right]
\end{array}\right]=\exp \left(i k_{0} d \sin \theta_{i}\right)\left[\begin{array}{l}
X\left(-\frac{r d}{2}\right) \\
V\left(-\frac{r d}{2}\right)
\end{array}\right] .
$$

we obtain an eigenvalue equation for $\Lambda^{2}\left(\Lambda^{2}\right.$ is contained in the definition of $\alpha$ and $\left.\beta\right)$ :

$$
0=\cos \left(k_{0} d \sin \theta_{i}\right)-\cos (\beta r d) \cos [\alpha(1-r) d]+\frac{1}{2}\left(\tau \frac{\alpha}{\beta}+\frac{1}{\tau} \frac{\beta}{\alpha}\right] \sin (\beta r d) \sin [\alpha(1-r) d],
$$

where $\tau=\epsilon$. For the $E$ polarization, if we write $E_{y}=X(x) \exp (i \Lambda z)$, then similar considerations yield exactly the same eigenvalue equation with $\tau=1$. To complete the solution, we write down the expression for $X$ :

$$
X(x)=\left\{\begin{array}{l}
\cos \left[\beta\left[x+\frac{r d}{2}\right)\right]+i V_{0} \frac{\tau k_{0}}{\beta} \sin \left[\beta\left(x+\frac{r d}{2}\right)\right], \quad|x| \leq \frac{r d}{2} \\
\left.U_{1} \cos \left[\alpha\left(x-\frac{r d}{2}\right)\right]+i V_{1} \frac{k_{0}}{\alpha} \sin \left[\alpha\left(x-\frac{r d}{2}\right)\right], \quad \frac{r d}{2} \leq|x| \leq \mid 1-\frac{r}{2}\right) d
\end{array}\right.
$$

where $X(-r d / 2)$ is normalized to 1 , with

$$
\begin{aligned}
V_{0}= & {\left[\exp \left(i k_{0} d \sin \theta_{i}\right)-M\right] / N } \\
V_{1}= & \frac{i}{k_{0}} \frac{\beta}{\tau} \sin (\beta r d)+V_{0} \cos (\beta r d), \\
U_{1}= & \cos (\beta r d)+i V_{0} \frac{\tau k_{0}}{\beta} \sin (\beta r d), \\
M= & \cos (\beta r d) \cos [\alpha(1-r) d] \\
& -\frac{\beta}{\tau \alpha} \sin [\alpha(1-r) d] \sin (\beta r d), \\
N=i k_{0} & {\left[\frac{1}{\alpha} \cos (\beta r d) \sin [\alpha(1-r) d]\right.} \\
& \left.\quad+\frac{\tau}{\beta} \sin (\beta r d) \cos [\alpha(1-r) d]\right],
\end{aligned}
$$

and

$$
\tau= \begin{cases}\epsilon & \text { for the } H \text { polarization } \\ 1 & \text { for the } E \text { polarization }\end{cases}
$$

The wave field in region II can therefore be ex- pressed in general as

$$
\Psi^{\mathrm{II}}=\sum_{l} X_{l}(x)\left[A_{l} \exp \left(i \Lambda_{l} z\right)+B_{l} \exp \left(-i \Lambda_{l} z\right)\right],
$$

where $l$ is the label for the eigenvalues, $X_{l}(x)$ is the eigenfunction associated with the eigenvalue $\Lambda_{l}$, and $\Psi$ has the same meaning as defined in Eqs. (1) and (2).

To gain a better grasp of the eigenvalue equation, we plot in Fig. 2 the right-hand side of Eq. (13), denoted by $F$, as a function of $\Lambda^{2}$ for a silver grating with $d=1050 \mathrm{~nm}, r=0.5$. The wavelength of the normally incident light is taken to be 700 $\mathrm{nm}$, and the dielectric constant of the silver at this wavelength is given by ${ }^{10} \epsilon=-23.4$ (for the sake of illustration, the small imaginary part is omitted to make the eigenvalue equation real). Three features of the figure should be noted. First, there is always a maximum value of $\Lambda^{2}$ (or a real part of $\Lambda^{2}$ for complex eigenvalues) that corresponds to the lowest eigenmode of the grating. Therefore, we can always order the eigenvalues according to the size of $\operatorname{Re}\left(\Lambda^{2}\right)$. Second, as $\Lambda^{2}$ decreases, there is 

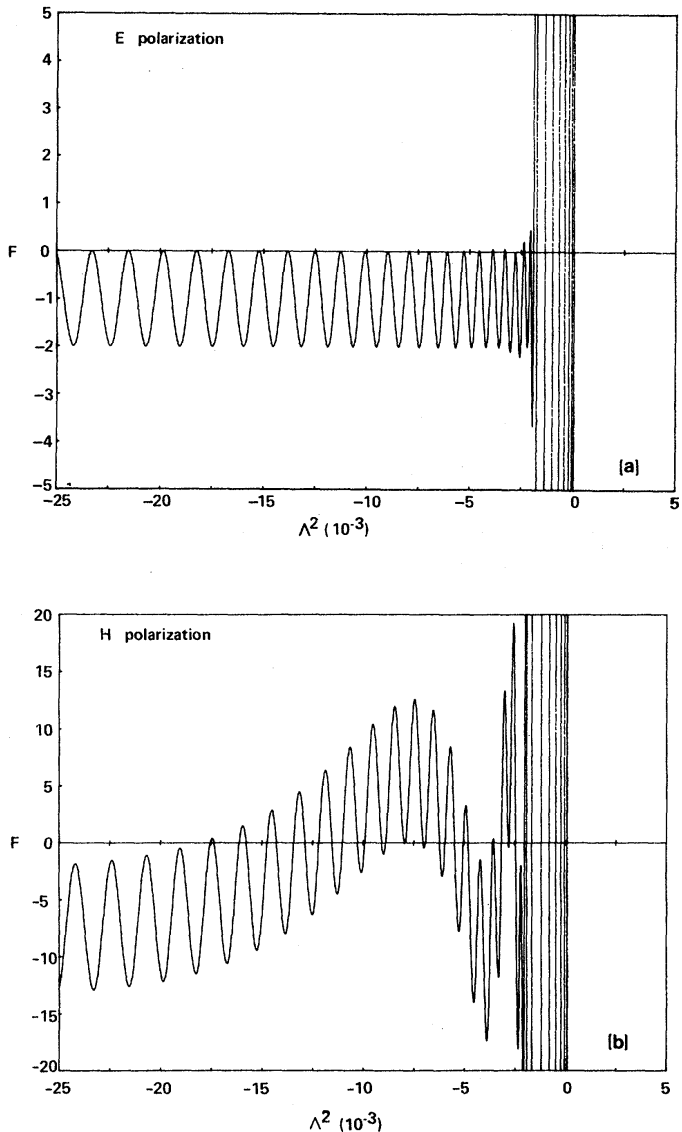

FIG. 2. Right-hand side of the eigenvalue equation, Eq. (13), plotted as a function of $\Lambda^{2}$. Each zero crossing denotes an eigenvalue. (a) $E$ polarization, (b) $H$ polarization.

an abrupt change in the character of $F$ from being exponentially large in amplitude to being relatively small in amplitude. This is due to the fact that $\alpha=\left(k_{0}^{2}-\Lambda^{2}\right)^{1 / 2}, \beta=\left(\epsilon k_{0}^{2}-\Lambda^{2}\right)^{1 / 2}$ switches from imaginary in value to real in value as $\Lambda^{2}$ decreases, thereby changing the nature of sine and cosine functions. Third, in the case of the $H$ polarization, the function $F$ is seen to cross zero on a regular basis only down to a certain value of $\Lambda^{2}$. Since zero crossings represent eigenvalues, at first sight it might seem that there is a gap in the eigenvalue spectrum at large negative values of $\operatorname{Re}\left(\Lambda^{2}\right)$. However, a closer examination shows that the eigenvalues just appear in complex conjugate pairs for those sections of $\operatorname{Re}\left(\Lambda^{2}\right)$ where $F$ does not cross 0 . [This can be understood by direct analogy with the quadratic equation where the parabola $y=f(x)$ does not cross the $x$ axis.]

For the same grating parameters and light wavelength as those in Fig. 2, the behavior of four eigenfunctions $X_{l}(x)$ are plotted in Figs. 3 and 4. It is seen that whereas the lower-order eigenfunctions show very little penetration into $\mathrm{Ag}$ and are therefore similar to those of the perfectly conducting grating (shown in dashed lines), the higher excited states exhibit significant amplitudes inside the metal.

\section{DIFFRACTION CALCULATION FORMALISM}

The analytic solution of Maxwell's equations in each of the three spatial regions has enabled us to expand arbitrary wave field $\Psi$ as a superposition of the eigenfunctions. The task of the diffraction calculation now is to explicitly determine the expan-
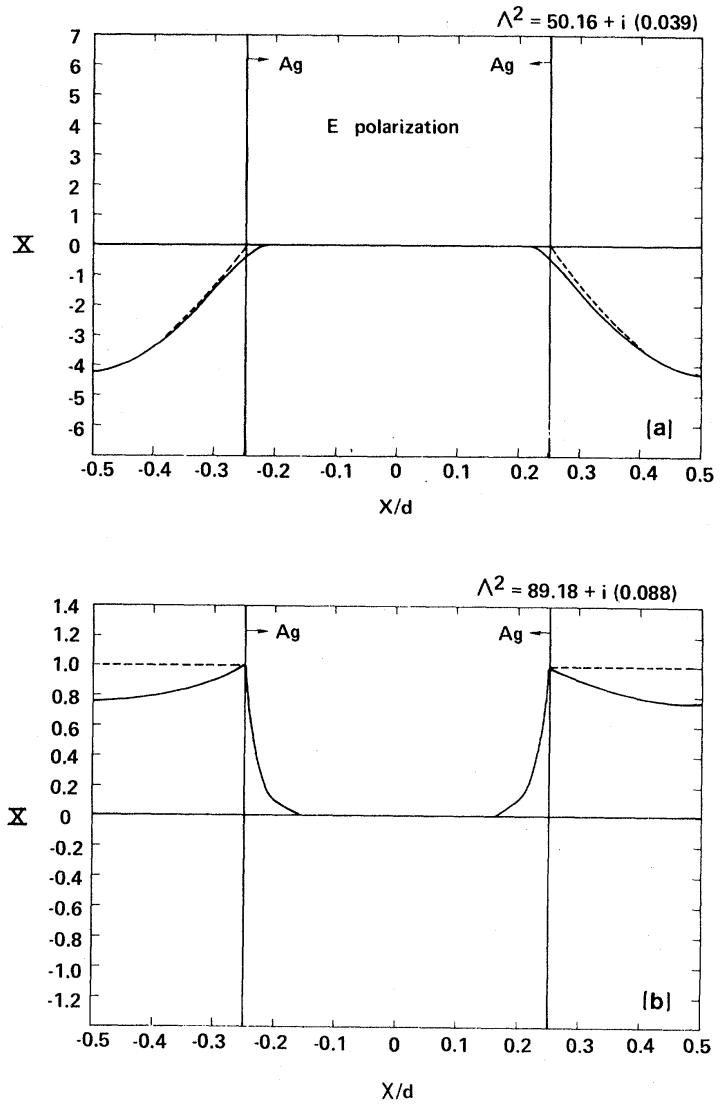

FIG. 3. Ground-state eigenfunctions $X(x)$ plotted as a function of $x / d$. The solid lines denote the real part of the eigenfunction. The dashed lines denote the corresponding eigenfunctions in the perfect conductor case. The imaginary parts of the ground-state eigenfunctions are small compared to the real parts and are now shown in the graph. (a) $E$ polarization, (b) $H$ polarization. $\theta_{i}=0^{\circ}$. 

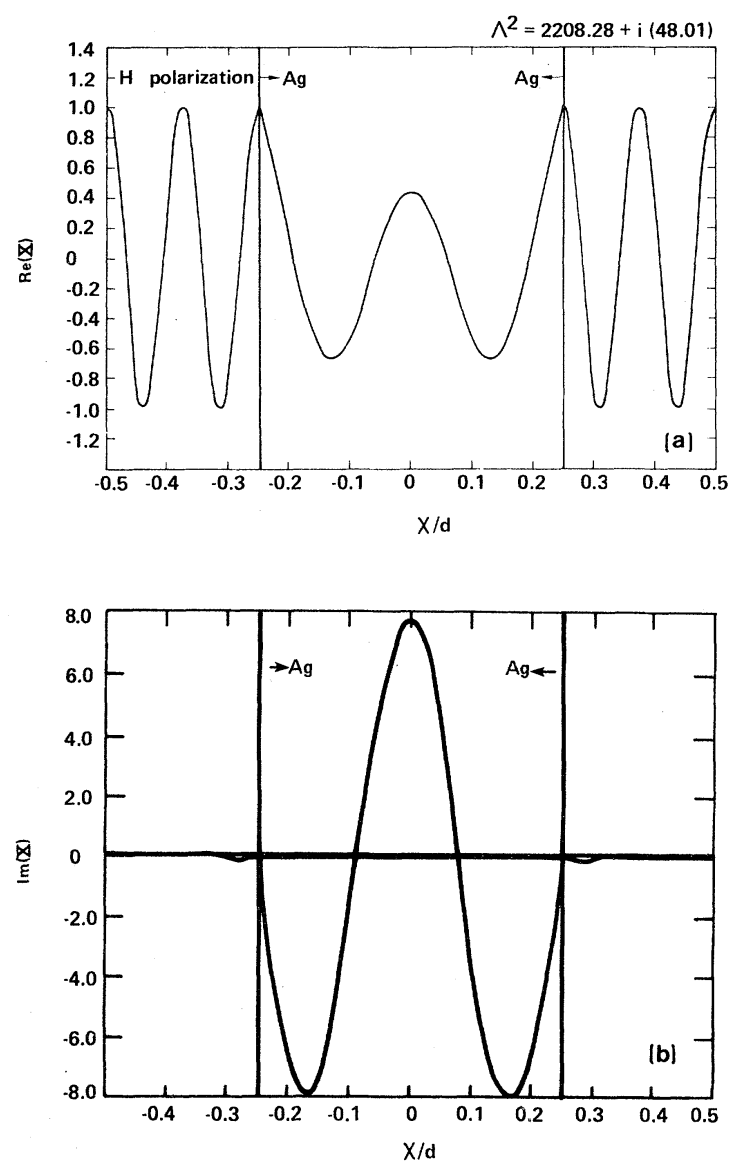

FIG. 4. Higher excited-state eigenfunction in the $H$ polarization. (a) The real part. (b) The imaginary part. Note the amplitude of the eigenfunction inside the metal. Similar behavior holds for the higher excited $E$ polarization eigenfunctions. $\theta_{i}=0^{\circ}$.

sion coefficients through the boundary conditions (continuity of tangential $E$ and $H$ fields) at $z=0$ and $z=-h$ :

$$
\begin{aligned}
& \Psi^{\mathrm{I}}(z=0, x)=\Psi^{\mathrm{II}}(z=0, x), \\
& \Psi^{\mathrm{II}}(z=-h, x)=\Psi^{\mathrm{II}}(z=-h, x), \\
& \frac{\partial \Psi^{\mathrm{I}}}{\partial z}(z=0, x)=\frac{\partial \Phi^{\mathrm{II}}}{\partial z}(z=0, x), \\
& \frac{\partial \Phi^{\mathrm{II}}}{\partial z}(z=-h, x)=\frac{1}{\tau} \frac{\partial \Psi^{\mathrm{III}}}{\partial z}(z=-h, x) .
\end{aligned}
$$

Here $\Phi^{\mathrm{II}}$ is defined as

$$
\Phi^{\mathrm{II}}=\sum_{l} W_{l}(x)\left[A_{l} \exp \left(i \Lambda_{l} z\right)+B_{l} \exp \left(-i \Lambda_{l} z\right)\right]
$$

with

$$
W(x)=\left\{\begin{array}{l}
\frac{1}{\epsilon(x)} X(x), \quad H \text { polarization } \\
X(x), \quad E \text { polarization }
\end{array}\right.
$$

Since Eqs. (22) - (25) are valid for all values of $x$, we can multiply each equation by $\exp \left(-i k_{0} \gamma_{m} x\right)$ and integrate over one period. This results in the following set of matrix equations:

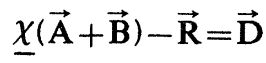

$$
\begin{aligned}
& \underline{\chi}\left(\underline{\Sigma} \overrightarrow{\mathrm{A}}+\underline{\Sigma}^{-1} \overrightarrow{\mathrm{B}}\right)-\underline{\Delta} \overrightarrow{\mathrm{T}}=0, \\
& \underline{\Omega}(\overrightarrow{\mathrm{A}}-\overrightarrow{\mathrm{B}})-\underline{\Pi} \overrightarrow{\mathrm{R}}=-\underline{\Pi} \overrightarrow{\mathrm{D}}, \\
& \underline{\Omega}\left(\underline{\Sigma} \vec{A}-\underline{\Sigma}^{-1} \overrightarrow{\mathbf{B}}\right)+\underline{\xi} \underline{\vec{T}}=0,
\end{aligned}
$$

where

$$
\begin{aligned}
& (\underline{\chi})_{m l}=\int_{\text {one period }} X_{l}(x) \exp \left(-k_{0} \gamma_{m} x\right) d x \\
& (\underline{\Omega})_{m l}=\Lambda_{l} \int_{\text {one period }} W_{l}(x) \exp \left(-i k_{0} \gamma_{m} x\right) d x \\
& (\underline{\Sigma})_{m l}=\exp \left(-i \Lambda_{l} h\right) \delta_{m l} \\
& (\underline{\Delta})_{m n}=\exp \left[i k_{0}\left(\epsilon-\gamma_{m}^{2}\right)^{1 / 2} h\right] \delta_{m n} \\
& (\underline{I})_{m n}=k_{0}\left(1-\gamma_{m}^{2}\right)^{1 / 2} \delta_{m n}
\end{aligned}
$$

and

$$
(\underline{\xi})_{m n}=\frac{k_{0}}{\tau}\left(\epsilon-\gamma_{m}^{2}\right)^{1 / 2} \delta_{m n}
$$

are the matrices, and $(\overrightarrow{\mathrm{A}})_{l}=A_{l},(\overrightarrow{\mathrm{B}})_{l}=B_{l}$, $(\overrightarrow{\mathrm{R}})_{m}=R_{m},(\overrightarrow{\mathrm{T}})_{m}=T_{m}$ are the unknown vectors. $\overrightarrow{\mathrm{D}}$ is defined as $(\overrightarrow{\mathrm{D}})_{m}=\delta_{m 0}$. Equations $(28)-(31)$ can easily be solved for $R$ and $T$. The results, in matrix form, are

$$
\overrightarrow{\mathbf{R}}=(\underline{\theta}-\underline{\Pi})^{-1}(\underline{\theta}+\underline{\Pi}) \overrightarrow{\mathrm{D}},
$$

with

$$
\begin{aligned}
\underline{\theta}=-\underline{\Omega} X^{-1}+ & 2 \underline{\Omega}\left(\underline{\Sigma}^{-1}+\underline{\Sigma}\right)^{-1} \\
& \times\left[\underline{\Omega}-\underline{\xi \chi}\left(\underline{\Sigma}^{-1}-\underline{\Sigma}\right)\left(\underline{\Sigma}^{-1}+\underline{\Sigma}\right)^{-1}\right]^{-1} \\
& \times(\underline{\Omega}-\underline{\xi}) \underline{\Sigma}^{-1} \underline{\chi}^{-1}
\end{aligned}
$$

and

$$
\overrightarrow{\mathrm{T}}=\underline{\Delta}^{-1} \underline{\chi}(\underline{I}+\underline{\Phi}) \underline{\Sigma}(\underline{I}+\underline{\Sigma \Phi \Sigma})^{-1} \underline{\chi}^{-1}(\overrightarrow{\mathrm{D}}+\overrightarrow{\mathrm{R}}),
$$

where 


$$
\Phi=(\underline{\Omega}-\underline{\xi \chi})^{-1}(\underline{\Omega}+\underline{\xi \chi}),
$$

and $\underline{I}$ is the identity matrix. It should be noted that in the composition of $\underline{\theta}$, the only matrix which depends on $h$ is $\underline{\Sigma}$. For $h=0, \underline{\Sigma}=\underline{I}$, and $\underline{\theta}$ matrix reduces to the simple form

$$
\left.\underline{\theta}\right|_{h=0}=-\underline{\xi} \text {, }
$$

which directly implies

$$
\begin{aligned}
& R=(\underline{\xi}+\underline{\Pi})^{-1}(\underline{\xi}-\underline{\Pi}) \overrightarrow{\mathbf{D}}, \\
& \overrightarrow{\mathbf{T}}=\underline{\Delta}^{-1}(\overrightarrow{\mathbf{D}}+\overrightarrow{\mathbf{R}}) .
\end{aligned}
$$

In the other limit of deep gratings, $h \rightarrow \infty$, we see that if $\Lambda_{l}$ has an imaginary part of any size, then $\underline{\Sigma} \rightarrow \infty \underline{I}$, which means $\underline{\theta}$ can again be reduced to a simple form:

$$
\left.\underline{\theta}\right|_{h \rightarrow \infty}=-\underline{\Omega} \underline{\chi}^{-1},
$$

and $T=0$. The fact that the $\underline{\theta}$ matrix, Eq. (38), interpolates between these two simple limits means that the deep gratings are just as easy to handle as flat surfaces in terms of numerical computation.

\section{NUMERICAL RESULTS AND COMPARISON WITH EXPERIMENTS}

Numerical calculations have been performed for both dielectric and metallic gratings. For the purpose of illustration, we will focus mainly on metallic gratings since traditionally they present more difficulties for numerical treatment. To carry out the computation, we first have to form the $\underline{\chi}$ and the $\underline{\Omega}$ matrices. Explicit expressions for the elements $(\underline{\chi})_{m l}$ and $(\underline{\Omega})_{m l}$ are given in the Appendix. Since truncation of the matrices is inevitable, care must be taken that the eigenfunctions whose eigenvalues form complex conjugate pairs do not get separated. The two eigenfunctions in the pair possess the same symmetry, and therefore should be considered as a single unit. To ensure the accuracy and reliability of the final solution, we have performed checks on the convergence of the amplitudes and energy conservation (the imaginary part of $\epsilon$ is set equal to zero for this check) by varying the rank of the $\underline{\theta}$ matrix.

In Figs. 5 and 6, we plot the (first-order) diffraction efficiency and absorption of $\mathrm{Ag}$ and $\mathrm{Al}$ gratings as a function of $h$ for both polarizations. The normally incident light is assumed to be at $\lambda=700$ $\mathrm{nm}$, and the gratings have the parameters $d=1050$ $\mathrm{nm}, r=0.5, \epsilon_{\mathrm{Ag}}=-23.4+0.387 i$, and ${ }^{10}$ $\epsilon_{\mathrm{Al}}=-42.6+17.02 i$. It is seen that whereas the
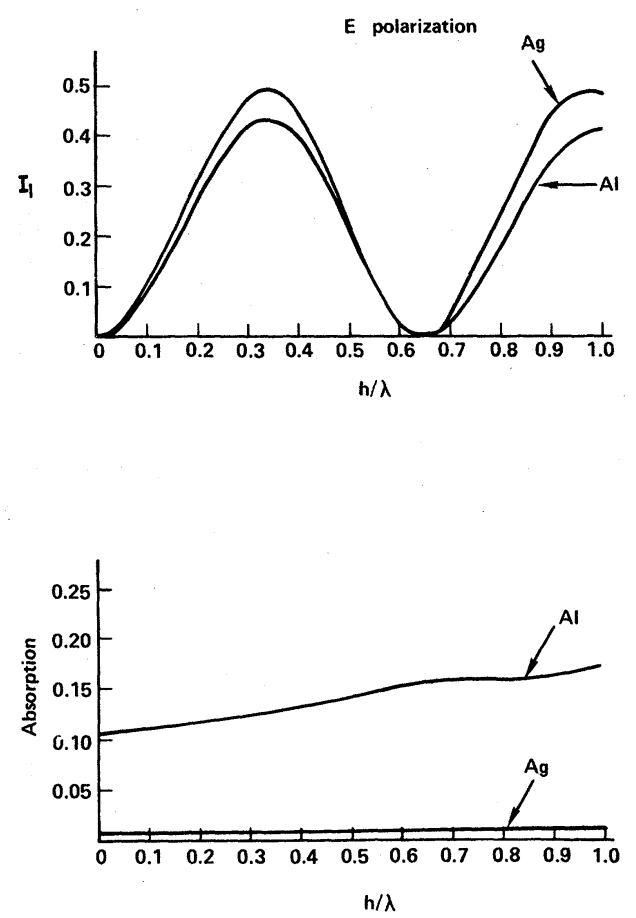

FIG. 5. First-order diffraction efficiency and total absorption plotted as a function of grating depth for normally incident $E$-polarized light with $\lambda=700 \mathrm{~nm}$.
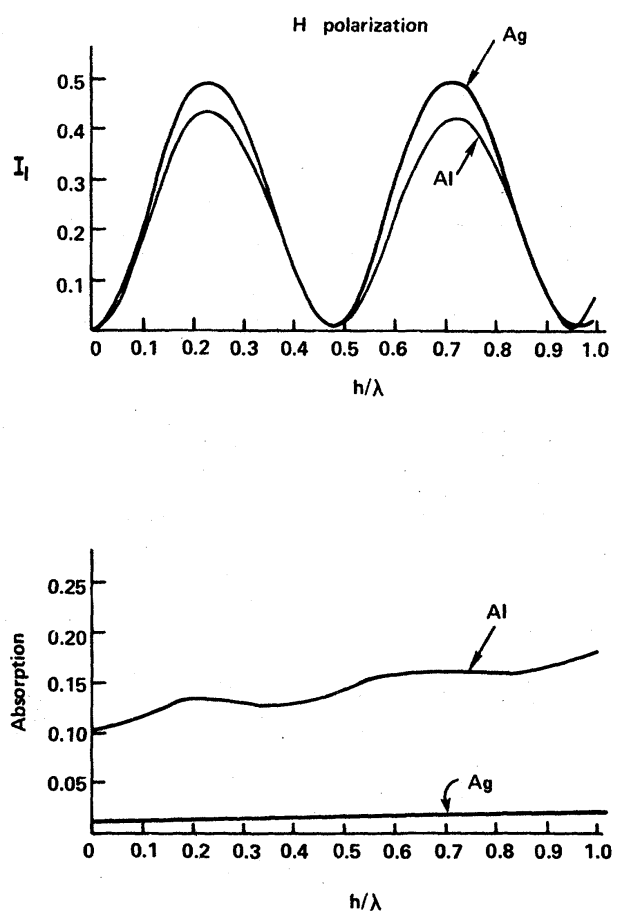

FIG. 6. First-order diffraction efficiency and total absorption plotted as a function of grating depth for normally incident $H$-polarized light with $\lambda=700 \mathrm{~nm}$. 
Ag grating acts as a nearly perfectly conducting grating, ${ }^{7}$ with little absorption at all values of $h$, the Al grating shows significant absorption with increasing depth. It should be remarked that the deep gratings presented no numerical difficulties.

A stringent test of the theory is the predictions of surface-plasmon excitations. Figure 7 shows the electron microscope picture of a square-wave $\mathrm{Ag}$ grating with $r=0.34, d=1 \mu \mathrm{m}$, and $h=0.06 \mu \mathrm{m}$. Using 6471- $\AA$ wavelength radiation from a krypton laser, we have measured the reflectivity as a function of $\theta_{i}$. The results are plotted in Fig. 8. For the $H$ polarization, the dips in the zeroth-order reflectivity correspond to the excitation of surface plasmon at the matching condition ${ }^{11}$

$$
k_{0} c=\omega\left(\left|k_{o} \sin \theta_{i}+(2 \pi n / d)\right|\right),
$$

where $c$ is the speed of light and $\omega(k)$ is the surface-plasmon dispersion relation. The reflectivity minima at $\theta_{i}=14^{\circ}, 25^{\circ}$, and $60^{\circ}$ correspond to $n=-1,1$, and -2 , respectively. It is seen that the theoretical curve, calculated by using $\epsilon_{\mathrm{Ag}}=-17.42+0.58 i$, agrees very well with the experimental data. In contrast to the $H$-polarization result, the $E$-polarization reflectivity shows a monotone behavior as shown in Fig. 9. Here the theoretical result again shows good agreement with experiment, even correctly predicting the small cusplike behavior at $\theta_{i}=20^{\circ}$.

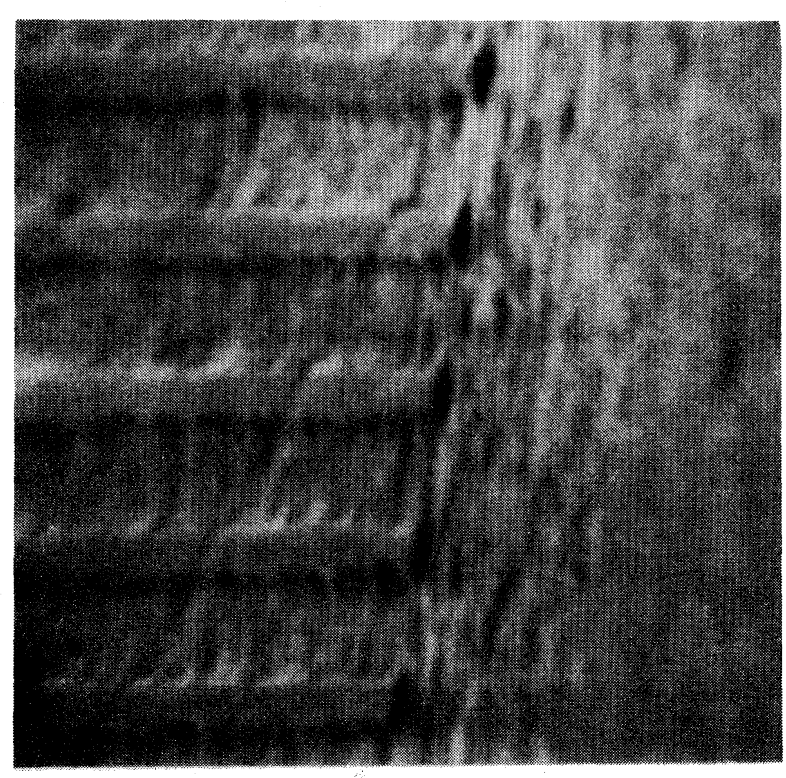

FIG. 7. Electron micrograph picture of a rectangular Ag grating. The parameters of the grating are given in the text.

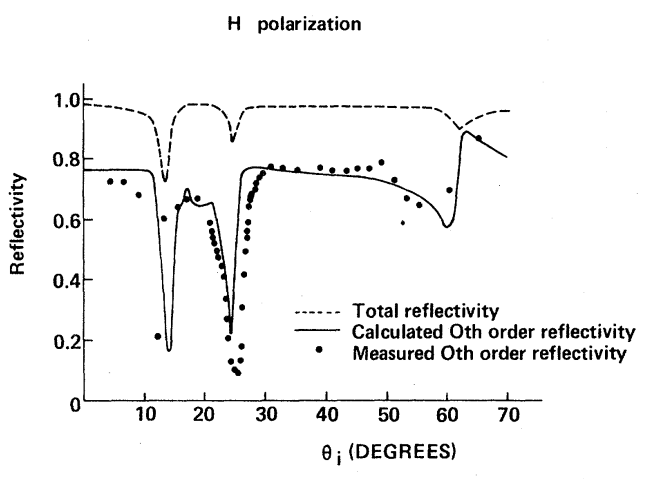

FIG. 8. Relectivity as a function of incident angle for the $H$ polarization. The dips are caused by the excitation of surface plasmons. Parameter values of the $\mathrm{Ag}$ grating are given in the text.

In Fig. 10, the electric field intensity at the surface of the grating is plotted for various incident angles. It is clear that as $\theta_{i}$ nears the excitation condition of the surface plasmon, the surface field intensity is increased by roughly a factor of 100 . Since local-field enhancement is believed to be responsible for a dominant part of the surfaceenhanced Raman scattering, our calculation indicates that the classical electromagnetic component of the enhancement factor can amount to $\sim(100)^{2}=10^{4}$. It must be emphasized, however, that we have only considered the bare grating without any molecular adsorbates. Therefore, the calculated surface field is just an order-ofmagnitude estimate of the actual local field seen by the absorbate molecules. Figure 11 illustrates the decay of the field intensity as a function of distance away from the grating surface. We can deduce from the graph that the characteristic de-

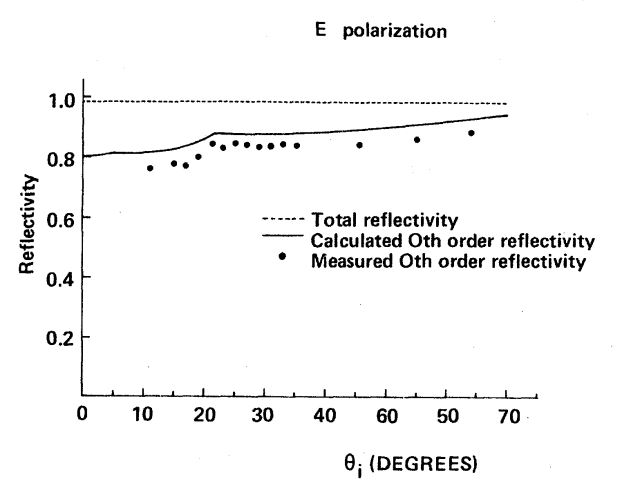

FIG. 9. Reflectivity as a function of incident angle for the $E$ polarization. The grating is the same as that of Fig. 8. 


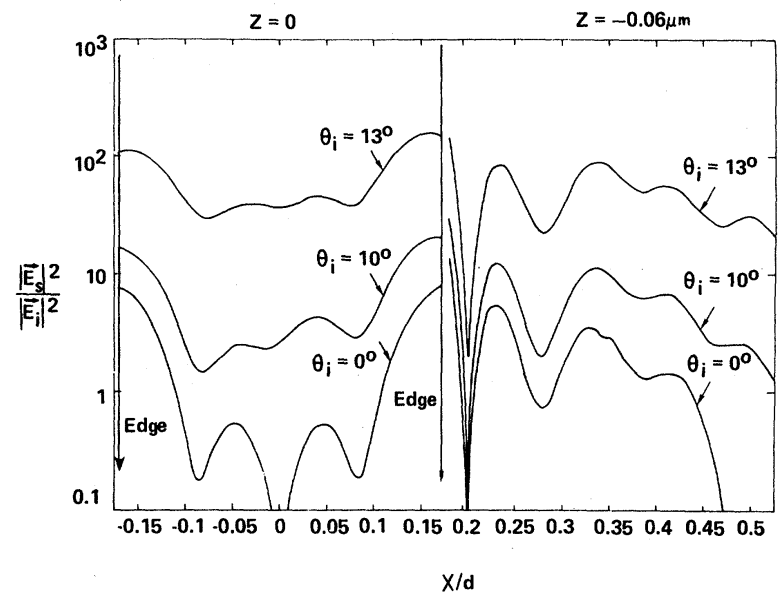

FIG. 10. Normalized electric field intensity at the grating surface. The grating parameters are the same as in Figs. 8 and 9. The edges of the $\mathrm{Ag}$ line are marked in the figure. The sharp corners cause the $|E|^{2}$ to increase toward the edges. Top of the grating corrugation is shown to the left; trough is shown to the right.

cay length of the resonance field is on the order of $1000 \AA$.

Another interesting phenomenon associated with the excitation of surface plasmons is the total absorption of $H$-polarized light by metallic gratings. ${ }^{12}$ A necessary condition for this occurrence is $d<\lambda$ so that the grating supports only the zeroth reflection order at normal incidence. Figure 12 shows that for an Ag grating with parameters $d=0.6$ $\mu \mathrm{m}, h=0.0105 \mu \mathrm{m}, r=0.5$, and $\epsilon_{\mathrm{Ag}}=-23.4+0.387 i$, an $H$-polarized incident wave with $\lambda=0.7 \mu \mathrm{m}$ is totally absorbed at $\theta_{i}=8.213^{\circ}$. It should be noted that the angular width of this total-absorption peak is extremely narrow, and a

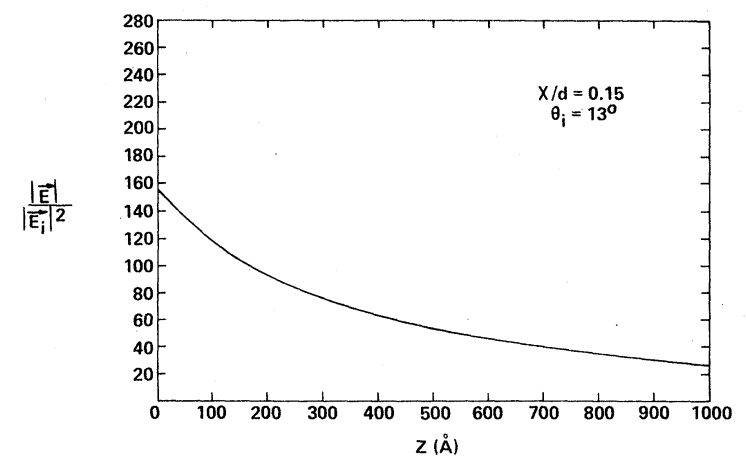

FIG. 11. Decay of the resonance-field intensity away from the surface of the grating. The grating parameters are the same as in Figs. 8 and 9.

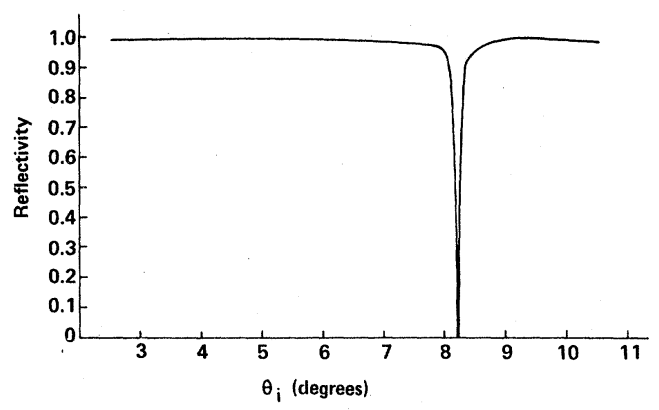

FIG. 12. $H$-polarization reflectivity as a function of incident angle $\theta_{i}$ : for a symmetric $\mathrm{Ag}$ grating with $d=0.6 \mu \mathrm{m}$ and $h=0.0105 \mu \mathrm{m}$.

-0.005 or a $+0.005 \mu \mathrm{m}$ deviation in $h$ from the value of $0.0105 \mu \mathrm{m}$ can raise the reflectivity dip from 0 to 0.2 or 0.7 , respectively. Such sensitivity to the $\theta_{i}, h$ parameters means that the phenomenon can be observed only under carefully controlled experiments. However, provided one can achieve these parameter values accurately, the field enhancement at the grating surface can be substantially larger than those shown in Fig. 9. In Fig. 13 , we plot the grating surface-field intensity at various $\theta_{i}$. It is seen that an increase of $\left|E_{s}\right|^{2} /\left|E_{i}\right|^{2}$ by a factor of $\geq 500$ is achieved, implying a Raman-enhancement factor of $\sim 3 \times 10^{5}$.

In summary, explicit photon eigenfunctions of the general square-wave grating have been derived. The use of these eigenfunctions in the diffraction calculation is shown to yield accurate predictions for the various types of grating phenomena. We have investigated the near-field electromagnetic properties of finite amplitude gratings in detail. The results indicate that the excitation of surface

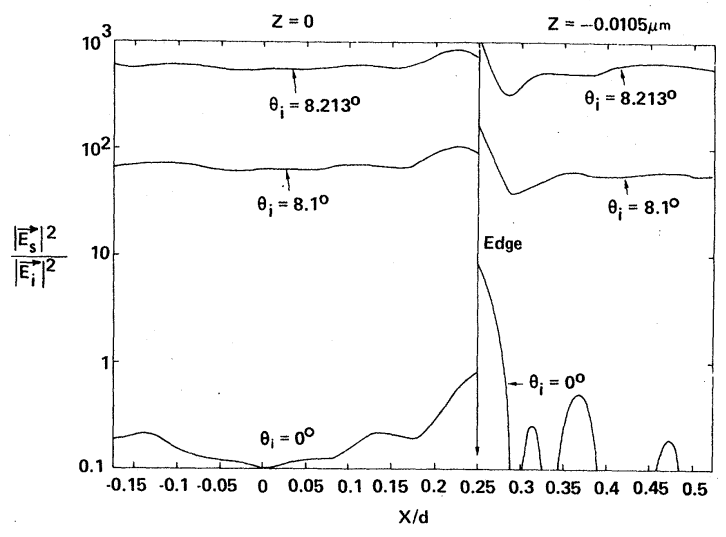

FIG. 13. Normalized electric field intensity at the grating surface. The grating parameters are the same as in Fig. 12. Top of the grating corrugation is shown to the left; trough is shown to the right. 
plasmon on Ag gratings coincides with a large enhancement of the near-surface-field intensity. Studies of coated gratings, as well as the calculation of emission pattern for an adsorbed dipole, are presently under way.

\section{ACKNOWLEDGMENTS}

One of (P.N.S) acknowledges J. M. Warlaumont for his collaboration in fabricating the Ag grating used in the reflectivity measurements and wishes to thank the staff of the IBM T. J. Watson Research Center for their assistance.

\section{APPENDIX}

Matrix elements $(\underline{\chi})_{m l}$ and $(\underline{\Omega})_{m l}$ can be obtained by direct integration of Eqs. (32) and (33). Dividing each of the integrals into two sections, one extending from $-d r / 2$ to $d r / 2$ and the other extending from $d r / 2$ to $(1-r / 2) d$, we immediately get

$$
\begin{aligned}
(\underline{\chi})_{m l}= & \exp \left(i k_{0} \gamma_{m} d r / 2\right)\left[\left[S_{m}^{+}(\beta)-T_{m}^{+}(\beta)\right]+V_{0} \frac{\tau k_{0}}{\beta}\left[S_{m}^{+}(\beta)+T_{m}^{+}(\beta)\right]\right) \\
& +\exp \left(-i k_{0} \gamma_{m} d r / 2\right)\left[U_{1}\left[S_{m}^{-}(\alpha)-T_{m}^{-}(\alpha)\right]+V_{1} \frac{k_{0}}{\alpha}\left[S_{m}^{-}(\alpha)+T_{m}^{-}(\alpha)\right]\right] \\
(\underline{\Omega})_{m l}= & \Lambda_{l} \exp \left(i k_{0} \gamma_{m} d r / 2\right)\left[\left[S_{m}^{+}(\beta)-T_{m}^{+}(\beta)\right]+V_{0} \frac{\tau k_{0}}{\beta}\left[S_{m}^{+}(\beta)+T_{m}^{+}(\beta)\right]\right) / \tau \\
& +\Lambda_{l} \exp \left[-i k_{0} \gamma_{m} d r / 2\right]\left[U_{1}\left[S_{m}^{1}(\alpha)-T_{m}^{-}(\alpha)\right]+V_{1} \frac{k_{0}}{\alpha}\left[S_{m}^{-}(\alpha)+T_{m}^{-}(\alpha)\right]\right) \\
S_{m}^{ \pm}(\beta)= & \frac{1}{2 i d} \frac{\exp \left\{-i\left(\beta-k_{0} \gamma_{m}\right) d[1 \pm(1-2 r)] / 2\right\}-1}{\beta-k_{0} \gamma_{m}}, \\
T_{m}^{ \pm}(\beta)= & \frac{1}{2 i d} \frac{\exp \left\{-i\left(\beta+k_{0} \gamma_{m}\right) d[1 \pm(1-2 r)] / 2\right\}-1}{\beta+k_{0} \gamma_{m}}
\end{aligned}
$$

The quantities $V_{0}, V_{1}, U_{1}$, and $\tau$ are, respectively, defined by Eqs. (15), (16), (17), and (20) in the text.

${ }^{1}$ See, for example, J. D. Jackson, Classical Electrodynamics (Wiley, New York, 1962), p. 297.

${ }^{2}$ Lord Rayleigh, Proc. R. Soc. London, Ser. A $\underline{79}, 399$ (1907).

${ }^{3}$ R. Petit and M. Cadilhac, C. R. Acad. Sci. 262B, 468 (1966).

${ }^{4}$ See, for example, Electromagnetic Theory of Gratings, edited by R. Petit (Springer, New York, 1980).

${ }^{5}$ N. Garcia, V. Celli, and N. R. Hill, Phys. Rev. B 18 , 5184 (1978).

6J. C. Tsang, J. R. Kirtley, and J. A. Bradley, Phys. Rev. Lett. 43, 772 (1979).

${ }^{7}$ P. Sheng, RCA Rev. 39, 512 (1978).

${ }^{8} \mathrm{M}$. Born and E. Wolf, Principles of Optics (Macmillan,
New York, 1964), p. 55.

${ }^{9}$ This condition is equivalent to the Bloch wave condition for electronic wave function in crystals.

${ }^{10}$ Optical data on $\mathrm{Ag}$ are obtained from P. B. Johnson and R. W. Christy, Phys. Rev. B 12, 4370 (1972); L. G. Shulz and F. R: Tangherlini, J. Opt. Soc. Am. 44, 357 (1954).

${ }^{11} \omega(k)$, can be obtained, for example, by treating $k_{0} \sin \theta$ as a complex variable and by locating the zeros of the matrix $(\underline{\theta}+\underline{\Pi})^{-1}(\underline{\theta}-\underline{\Pi})$. Such zeros correspond to the condition of resonance in the absence of incident wave [see Eq. (43)].

12D. Maystre and R. Petit, Opt. Commun. 17, 196 (1976). 


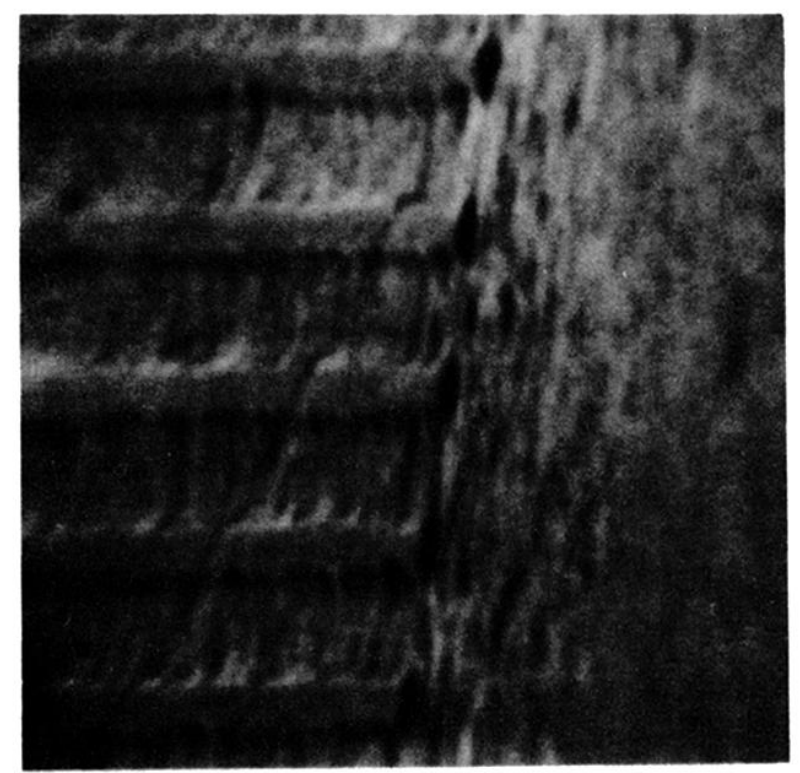

FIG. 7. Electron micrograph picture of a rectangular $\mathrm{Ag}$ grating. The parameters of the grating are given in the text. 